\title{
Predicción de riesgo ecotoxicológico dada la exposición a cianuro libre mediante modelación cinético-matemática en condiciones controladas utilizando el biomonitor Gambusia punctata
}

Ecotoxicological risk prediction given the free cyanide exposure by modeling kinetic -mathematical under controlled conditions using the BioMonitor Gambusia punctata

Recibido: mayo de 2016 | Revisado: junio de 2016 | Aceptado: junio de 2016

Ruben Ticona Huayhua ${ }^{\mathrm{I}}$ George Argota Pérez ${ }^{2}$
1 Facultad Físico-Matemática. Universidad Nacional del Altiplano, Puno-Perú. rubenspuno@hotmail.com

2 Centro de Investigaciones y Formación Superior en Educación, Salud y Medio Ambiente "AMTAWI“. Perú.

george.argota@gmail.com

\begin{abstract}
The goal of the study was to predict the ecotoxicological risk exposure given free cyanide by kinetic- mathematical modeling under controlled conditions using the ecotoxicological BioMonitor Gambusia punctata. The study was conducted in April 2016. Water samples from three different sources from the ecosystem - Ramis River Basin Titicaca in Puno, Peru were taken. Previously, the free cyanide concentration was determined using the $\mathrm{P}$ silver nitrate method and in laboratory conditions. Subsequently, the median lethal toxicity test with exposure time was carried out through 96 hours of experimentation with BioMonitor Gambusia punctata where the ecotoxicological prediction was made by kinetic-mathematical modeling of free cyanide exposure in experimental conditions. It was found that the concentrations of free cyanide at the sampling points were among 014 to $0.16 \mathrm{mg} / \mathrm{L}$ - 1, which exceeded recommended by the environmental standard used to set a maximum allowable limit of 0.022 . It was also noted that depending on the dose-response curve, total mortality of individuals 24 hours after taking the test, where it was observed that at a concentration of $0.078 \mathrm{ppm}$, equivalent to $50 \%$ dilution produced $50 \%$ of death in individuals. The regression equation between mortality and time showed that a concentration of $0.024 \mathrm{ppm}$ for 20 minutes caused the death of $10 \%$ of individuals. It was concluded that the free cyanide is found in bioavailable form that and that its concentrations, even being low showed effects of ecotoxicological risk, causing death in a relatively short time.
\end{abstract}

Keywords: ecotoxicological risk prediction, free cyanide, kinetic - mathematical modeling, bioassay, bioMonitor, Gambusia punctata, Puno - Peru

\section{RESUMEN}

El objetivo del estudio fue predecir el riesgo ecotoxicológico dada la exposición a cianuro libre mediante modelación cinético-matemática en condiciones controladas utilizando el biomonitor ecotoxicológico Gambusia punctata. El estudio se realizó en abril de 2016. Se tomaron muestras de aguas procedentes de tres puntos correspondientes al ecosistema Ramis-Cuenca Hidrográfica Titicaca en Puno, 
Perú. Previamente, se determinó la concentración libre de cianuro libre mediante el método $\mathrm{P}$

Pde nitrato de plata y en condiciones de laboratorio. Posteriormente, se realizó la prueba de toxicidad letal media con tiempo de exposición durante 96 horas de experimentación con el biomonitor Gambusia punctata donde se efectuó la predicción ecotoxicológica mediante modelación cinético-matemático de la exposición a cianuro libre en condiciones experimentales. Se encontró que las concentraciones de cianuro libre en los puntos de muestreo, estuvieron entre $014-0.16 \mathrm{mg} / \mathrm{L}^{-1}$, lo cual superó lo recomendado por la norma ambiental utilizada que establece un límite máximo permisible de 0.022 . Se observó según la curva dosis-respuesta, mortalidad total de los individuos a las 24 horas de duración del ensayo, donde se observó que a una concentración de $0.078 \mathrm{ppm}$, equivalente al $50 \%$ de dilución se produjo el $50 \%$ de muerte en los individuos. La ecuación de regresión entre la mortalidad y el tiempo, arrojó que una concentración de $0.024 \mathrm{ppm}$ durante 20 minutos, ocasionó la muerte del $10 \%$ de los individuos. Se concluyó que el cianuro libre está en forma biodisponible en que sus concentraciones aun siendo bajas presentaron efectos de riesgo ecotoxicológico, provocando la muerte en un tiempo relativamente corto.

Palabras claves: predicción de riesgo ecotoxicológico, cianuro libre, modelación cinético-matemática, bioensayo, biomonitor, Gambusia punctata, Puno-Perú

\section{Introducción}

El riesgo ha sido definido de diversas maneras con relación al ámbito como a la exposición; y donde Hogarth (2006), define al riesgo como una actividad que puede tener dos componentes: la posibilidad o probabilidad de que un resultado negativo ocurra y el tamańo de ese resultado; por cuanto, mientras mayor sea la probabilidad y la pérdida potencial, mayor será el riesgo.

En el caso particular de los estudios ambientales, Montico y Di Leo (2015), mencionan que, actualmente, se aplican indicadores con una dimensión ecotoxicológica para visualizar el impacto ambiental, peligrosidad además del posible riesgo a la salud, ya sean de manera simples o complejos, multicriterios y de criticidad entre otros y donde finalmente, ello posibilitará tomar las decisiones.
Por su parte, la United States Environmental Protection Agency (US EPA, 2001), definió que en el campo de la salud y del medio ambiente, el riesgo se identifica como la probabilidad de que un individuo o una población presenten una mayor incidencia de efectos adversos por exposición a un peligresgo. La Academia Nacional de Ciencias de los Estados Unidos (NAS, 1983), refirió que la identificación del peligro, es el proceso de determinar si un compuesto químico está vinculado con ciertos efectos a la salud como pueden ser el desarrollo de cáncer o defectos en el desarrollo.

Whyte y Burton (1980) mencionaron que el riesgo tiene dos significados distintos, uno en el contexto de peligro y otro de probabilidad o cambio de sufrir una consecuencia adversa. En el caso de una evaluación de riesgo ambiental, la US EPA (1998) divide a la misma en tres lineamien- 
PREDICCIÓN DE RIESGO ECOTOXICOLÓGICO DADA LA EXPOSICIÓN A CIANURO LIBRE MEDIANTE MODELACIÓN

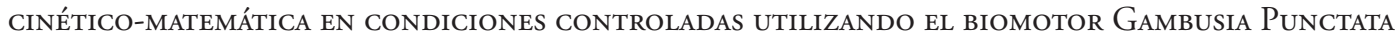

tos que comprende las siguientes etapas: (a) definición del problema, (b) análisis que comprende la caracterización de la exposición y de los efectos ecológicos y; (c) la caracterización del riesgo (Lee-Steere, 2009; United Nations Environment Programme and International Programme on Chemical Safety, 1999).

Según Morgan y Henrion (1990), las evaluaciones de riesgo proporcionaban un valor simple como una estimación conservadora del riesgo, mientras que hoy se acepta por lo general; que en la caracterización del riesgo, se requiere proporcionar un mayor entendimiento de los métodos de estimación y de la incertidumbre involucrada en la estimación.

Carpenter (1995) señaló que la evaluación de riesgos ambientales (ERA) sigue las reglas de la teoría de la probabilidad mediante una expresión de todos los posibles valores de cada parámetro analizado. Management Programme for the Gorgon Development (MPGD, 2004) indicó que la ERA es un proceso que evalúa la probabilidad y consecuencia de un impacto adverso que ocurre como resultado de la exposición a uno o más estresores.

Van der Oost, Beyer y Vermeulen (2003) argumentaron que deberían analizarse los biomarcadores (proteínas de estrés, metalotioneínas, parámetros hematológicos, parámetros inmunológicos, parámetros genotóxicos, parámetros endocrinos, etc.) y marcadores de bioacumulación en peces (xenobióticos: contaminantes orgánicos persistentes) y su empleo en ERA (procedimiento por el cual probable o real un efecto adverso de un contaminante y otras actividades antropogénicas en el ecosistema y sus componentes son estimados con un grado de certidumbre usando metodología científica). Independientemente de lo comentado anteriormente, se considera que las evaluaciones de toxicidad aguda (corto plazo) con mode- los biológicos de experimentación, del mismo modo resultan de relevancia para indicar determinadas probabilidades de riesgo, aunque siempre deberá considerarse el nivel de incertidumbre que cada exposición presenta de forma implícita.

En cuanto a la incertidumbre en la evaluación de riesgos, puede originarse por distintas causas como son la falta de información, diferencias en el nivel de la evidencia, simplificaciones o suposiciones realizadas para hacer factible el análisis, siendo importante distinguir entre incertidumbre y variabilidad, debido a que ambas pueden ocasionar incertidumbres en los resultados de la evaluación de riesgo (Frey, 1992).

Darbra, Eljarrat y Barceló (2008) indicaron que la incertidumbre en la evaluación de riesgos puede tener dos orígenes: aleatorización e incompletitud, existiendo dos formas principales: (1) la teoría de la probabilidad y (2) la lógica difusa. La incertidumbre es generada sobre el conocimiento incompleto del valor verdadero de un parámetro y se origina en la necesidad de establecer inferencias para pequeńas muestras sobre los procesos que tienen un cierto grado de aleatoriedad (Evans, 2002).

El propio Morgan y Henrion (1990), sostienen que la visión subjetiva de la probabilidad es el grado de confiabilidad que una persona tiene de que ocurrirá un evento tomando en cuenta, toda la información disponible y conocida por la persona. La probabilidad no solo depende del evento en sí, sino del nivel de información disponible acerca del mismo.

Rao et al. (2012) expresaron que las incertidumbres involucradas con la evaluación de riesgo son inherentes a la propia metodología y con frecuencia pueden complicar las decisiones. Sin embargo, muchas de estas incertidumbres pueden reducirse mediante la investigación. Así, los tomadores de deci- 
siones se enfrentan con el dilema de actuar de inmediato, basándose en los niveles presentes de información incierta o de esperar para obtener mayores evidencias y tomar así mejores decisiones en el futuro.

La evaluación de riesgos no necesariamente requiere la aplicación de técnicas sofisticadas o la recolección excesiva de datos. Se pueden obtener resultados prácticos y razonables utilizando información mínima disponible sobre la contaminación y sobre la población expuesta a ella (Lee-Steere, 2009 World Bank [WB], United Nations Environment Programme \& the United $\mathrm{Na}$ tions Industrial Development Organization, 1999).

Es importante, destacar que el pasado siglo XX y lo que transcurre en este siglo XXI, es cada vez más acuciante la contaminación ambiental como una de las situaciones ambientales globales (Samantray, Mishra, Panda y Rout, 2009). En tal sentido, una de las actividades que más contaminación está generando es la minería aurífera, donde la utilización del cianuro resulta altamente tóxico cuando es descargado a los cuerpos de aguas superficiales; $y$ en que son limitados los estudios que indican por una parte, cuáles son las concentraciones mínimas que evidencian efectos adversos y por otra parte, en qué tiempo dichas concentraciones comienzan a provocar estos efectos adversos. El objetivo del estudio fue predecir el riesgo ecotoxicológico dada la exposición a cianuro libre mediante modelación cinético-matemática en condiciones controladas utilizando el biomonitor ecotoxicológico Gambusia punctata.

\section{Método}

\section{Objeto de investigación y periodo de es- tudio}

Se estudió el comportamiento cinético-matemático de la toxicidad del cia- nuro libre en condiciones controladas de laboratorio. Para ello, se utilizaron muestras de aguas las cuales procedieron de tres puntos ambientales de selección, cuya posición de coordenadas georreferenciadas fueron: Punto A (latitud 14036'92' S, longitud $70^{\circ} 19^{\prime} 17^{\prime \prime} \mathrm{O}$ ), Punto B (latitud $140^{\circ} 20^{\prime} 44.32^{\prime \prime} \mathrm{S}$, longitud 700 $0^{\prime} 57.76^{\prime \prime} \mathrm{O}$ ) y Punto C (latitud 14040'25.91''S, longitud 69031'43.35'”). Estas coordenadas correspondieron al ecosistema Ramis-Cuenca Hidrográfica Titicaca en Puno, Perú. El estudio se realizó en el mes de abril de 2016.

\section{Muestra}

Se tomaron tres litros de agua por puntos de muestreo, los cuales fueron posteriormente homogenizados para su representación según el punto de selección en relación con el valor de uso del recurso agua. Las muestras se recogieron con cuidado de la capa superficial sin remover el sedimento, fundamentalmente, en lugares poco profundos. El volumen de muestra tomada en cada estación fue aproximadamente de 5 litros (ISOa,b,c). Luego, las muestras se homogeneizaron en un tanque de polietileno previamente endulzado con las propias aguas del río. Se realizó un muestreo probabilístico aleatorio simple, donde se tuvo en cuenta la misma frecuencia horaria y tiempo de permanencia de muestra para minimizar los errores aleatorios asociados al muestreo. Cada punto de muestreo fue previamente identificado con la finalidad de conservar la fidelidad de los datos.

Una vez realizados los muestreos, las muestras de agua fueron depositadas en acuatorios por 24 horas para su posterior experimentación con el monitor ecotoxicológico Gambusia punctata.

Los métodos cuantitativos de investigación aplicados fueron el teórico de la modelación, empírico de medición, así como el experimental de enfoque. 
PREdicCión DE RIESGo ECOTOXICOLÓGICO DADA LA EXPOSICIÓN A CIANURO LIBRE MEDIANTE MODELACIÓN

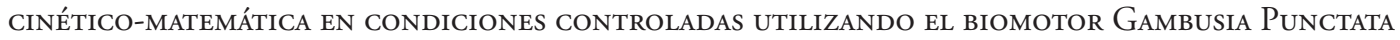

\section{Análisis de las variables}

\section{Determinación en efluentes de cianuro libre. Método del nitrato de plata}

\section{I.1 Fundamento}

El método de determinación de cianuro libre $\left(\mathrm{CN}^{-}\right)$por valoración con $\mathrm{AgNO}_{3}$, se basa en la formación del ión complejo $\mathrm{Ag}(\mathrm{CN})_{2}$ - el cual es muy estable e incoloro.

- $2 \mathrm{CN}^{-}+\mathrm{Ag}^{+} \mathrm{NO}_{3}^{-} \longrightarrow \mathrm{Ag}^{-}(\mathrm{CN})_{2}^{-}$

- $\mathrm{AgNO}_{3}+2 \mathrm{NaCN} \rightarrow \mathrm{NaAg}(\mathrm{CN})_{2}+\mathrm{NaNO}_{3}$

$\mathrm{Al}$ acomplejarse todo el ión cianuro presente y existe un pequeño exceso de $\mathrm{Ag}+$ ańadido, este exceso es detectado por el indicador 5-(4-dimetilaminobencilideno)-Rodanina, que es sensible a la plata y cambia inmediatamente de color amarillo a color anaranjado.

Luego, a medida que se añade nitrato de plata (gota a gota desde la bureta) se consume el cianuro en disolución para formarse $\mathrm{NaAg}(\mathrm{CN})_{2}$. Cuando el cianuro presente en la disolución se consume, la plata formada con el nitrato, comienza a reaccionar con las gotas de rodanina añadidas, formando un compuesto rosado.

\section{I.2 Procedimiento}

Si hay sulfuros en la muestra, añadir $5 \mathrm{ml}$ de la solución $0.5 \mathrm{M}$ de nitrato de cadmio preparada previamente. Luego dejar sedimentar el precipitado amarillo de sulfuro de cadmio y filtrar sin remover el precipitado hasta el final, a través de un filtro de papel de porosidad media. Lavar el papel y el residuo con agua caliente dos veces.

Posteriormente, añadir al filtrado $0.5 \mathrm{ml}$ de la solución del indicador Rodanina. Valorar con la solución estándar de nitrato de plata hasta que el color amarillo cambie hasta anaranjado. Para confirmar el cambio, valorar paralelamente una solución en blanco que contenga las mismas cantidades de agua e indicador que las utilizadas en la muestra.

\section{I.3 Expresión de resultados}

$$
\mathrm{NaCN}(\mathrm{ppm})=(\mathrm{a}-\mathrm{b}) / \mathrm{V} \text { alícuota } * 1000
$$

\section{Determinación de la concentración de toxicidad letal media por exposición a cianuro libre en el biomonitor ecotoxi- cológico Gambusia punctata.}

Para las baterías de ensayo en el biomonitor ecotoxicológico Gambusia punctata, pudo seguirse lo recomendado por la Organización de Cooperación y Desarrollo Económico (OECD, 1992), estableciéndose para ello, cinco réplicas por el tratamientos incluyendo un control negativo.

\section{II.1 Principio del ensayo}

Durante el ensayo se expondrán individuos alevines y juveniles seleccionados a diferentes concentraciones de muestras del problema (aguas del río Ramis). Este ensayo será utilizado para determinar efectos tóxicos agudos ocasionados por las aguas. Este método de prueba es semejante al protocolo de la Agencia para la Protección Ambiental de los Estados Unidos de Norteamérica (US EPA, 2002).

\section{II.2 Condiciones experimentales}

Los individuos se aclimataron 24 horas antes del estudio en acuatorios de cristal y sin alimentación durante el período de ensayo.

La temperatura del ensayo fue controlada y climatizada a $18^{\circ} \mathrm{C}$. El período de prueba consistió en 96 horas, donde el parámetro a medir fue la concentración letal $50\left(\mathrm{CL}_{50}\right)$ como toxicidad aguda. El análisis estadístico para medir la $\mathrm{CL}_{50}$ será el método gráfico de Litchfield y Wilconxon (1949), que establece básicamente la curva dosis-respuesta.

Los datos de concentración/mortalidad se utilizan para calcular la concentración letal 
media o $\mathrm{CL}_{50}$, la cual se puede determinar por el método Probit o Trimmed-Spearman, según sea el caso. Para ello, se utilizó el software proporcionado por la USEPA, que describe lo siguiente:

$$
\mathbf{p}=(\mathrm{r} / \mathrm{n}) \times 100
$$

Donde:

- Número de individuos (n),

- Número de organismos muertos o afectados $(r)$.

- Porcentaje de efecto $(p)$.

La representación gráfica de $p$ s. $d$, o relación dosis-respuesta, genera una curva parabólica que muchas veces presenta dificultades en la construcción de un modelo lineal. Una forma de abordar este problema es transformando $d$ a una escala logarítmica $(X$ $=\log 10(d)$, que mostrará una relación dosis-respuesta de forma $S$ o sigmoidea normal.

Posteriormente, mediante las Tablas de Probit se transforma $p$ (porcentaje de efecto) a unidades probit (buscando en una tabla de distribución normal el valor de $z$ correspondiente a una probabilidad acumulada igual a $p$ y sumándole a continuación cinco unidades), se obtiene una distribución de puntos en un sistema bivariado de tipo lineal, que se procesan según un análisis de regresión típica. Vale la pena enfatizar en que el Probit es una transformación sobre la tasa de efecto $(p)$ y la ecuación generada es de la forma:

$\mathbf{y}=a+b x$

Donde:

- $\quad \mathrm{y}$ (expresado en unidades probit $)=\mathrm{z}$ $+5$

- $\mathrm{z}=$ variable normal estándar $=\mathrm{z}_{0}$ tal que la Prob $\left(\mathrm{z} \leq \mathrm{z}_{0}\right)=\mathrm{p}$

- a y b son los estimadores de los parámetros de la recta de regresión
- Cuando $\mathrm{p}=50 \%$ entonces $\mathrm{y}=5$, por tanto: $\mathrm{x}_{5}=\log 10 \mathrm{CL}_{50}=10 \mathrm{X5}$

\section{Tratamiento estadístico de los datos}

Para el tratamiento de los resultados se aplicaron métodos estadísticos, los cuales correspondieron al análisis de la varianza factorial con réplicas para definir las fuentes de variación significativas y la prueba de intervalos múltiples de Duncan para determinar las magnitudes individuales de las diferencias que resulten significativas según lo expresado por Montgomery (1991). Todos los cálculos se realizaron utilizando el software profesional Statgraphics (Statpoint Technologies, 1994-2001), en que los resultados se consideraron significativos a un nivel de confianza del $95 \%(\mathrm{p} \leq 0.05)$.

\section{Resultados}

Dadas las determinaciones de cianuro libre $(0.012$ a $0.078 \mathrm{ppm})$ que previamente se conocía (Argota et al., 2015), fueron ensayadas las mismas en condiciones de laboratorio. En la Tabla 1, pueden mostrarse las variables que definen la toxicidad letal por exposición a cianuro libre en las aguas, así como los análisis estadísticos correspondientes (Tabla 2 y 3 ).

Tabla 1

Variables que definen la toxicidad letal por exposición a cianuro libre en las aguas

\begin{tabular}{ccc}
\hline $\begin{array}{c}\text { Tiempo } \\
(\mathbf{m i n})\end{array}$ & $\begin{array}{c}\text { Concentración } \\
\left(\mathbf{m g} / \mathbf{L}^{-1}\right)\end{array}$ & $\begin{array}{c}\text { Mortalidad } \\
(\%)\end{array}$ \\
\hline 10 & 0.012 & 0 \\
15 & 0.018 & 0 \\
20 & 0.024 & 10 \\
25 & 0.030 & 30 \\
30 & 0.036 & 50 \\
35 & 0.042 & 60 \\
40 & 0.048 & 70 \\
45 & 0.057 & 80 \\
50 & 0.063 & 80 \\
55 & 0.070 & 90 \\
60 & 0.078 & 100 \\
\hline
\end{tabular}

Nota: Elaboración propia (2016). 
PREdicCión DE RIESGo ECOTOXICOLÓGICO DADA LA EXPOSICIÓN A CIANURO LIBRE MEDIANTE MODELACIÓN

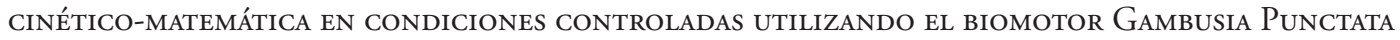

Tabla 2

Análisis de significación para la mortalidad

\begin{tabular}{ccccc}
\hline \multicolumn{5}{c}{ Variable dependiente: mortalidad } \\
\hline parámetro & error estimación & error estándar & $\mathrm{t}_{\text {calculada }}$ & P-valor \\
\hline constante & -33.129 & 6.1418 & -5.39402 & 0.0007 \\
concentración & -3798.45 & 1715.96 & -2.21359 & 0.0578 \\
tiempo & 7.14306 & 2.25254 & 3.17111 & 0.0132 \\
\hline
\end{tabular}

Nota: Elaboración propia (2016). Análisis en el programa estadístico Statgraphisc Plus 5.1.

Tabla 3

Análisis de la varianza con tres grupos

\begin{tabular}{cccccc}
\hline Fuente & $\begin{array}{c}\text { Suma de } \\
\text { cuadrados }\end{array}$ & $\begin{array}{c}\text { Grados de } \\
\text { libertad }\end{array}$ & $\begin{array}{c}\text { Cuadrado } \\
\text { medio }\end{array}$ & F - calculado & P-valor \\
\hline Modelo & 13059.8 & 2 & 6529.88 & 171.91 & 0.0000 \\
Residuo & 303.876 & 8 & 37.9845 & & \\
Total (Corr.) & 13363.6 & 10 & & & \\
\hline
\end{tabular}

Nota: Elaboración propia (2016). Análisis en el programa estadístico Statgraphisc Plus 5.1.

- $\quad$ R-cuadrado $=97.7261$ porcentaje

- R-cuadrado (ajustado para g.l.) = 97.1576 porcentaje

- $\quad$ Error estándar de est. $=6.16316$

- $\quad$ Error absoluto medio $=4.46281$

- Estadístico de Durbin-Watson = $1.82384(\mathrm{P}=0.1324)$

- Autocorrelación residual en Lag 1 = $-0.00023616$

La salida muestra los resultados del ajuste a un modelo de regresión lineal múltiple para describir la relación entre mortalidad y dos variables independientes (concentración y tiempo). La ecuación del modelo ajustado es:

Mortalidad $=-33.129-$ $3798.45 *$ concentración $+7.14306 *$ tiempo

Dado que el p-valor en la tabla ANOVA fue inferior a 0.01 , existe relación estadísti- camente significativa entre las variables para un nivel de confianza del $99 \%$.

El estadístico R-cuadrado indica que el modelo explica un $97.7261 \%$ de la variabilidad en mortalidad. El estadístico R-cuadrado ajustado, que es más conveniente para comparar modelos con diferente números de variables independientes, es $97.1576 \%$. El error estándar de la estimación muestra la desviación típica de los residuos que es 6.16316. El error absoluto medio (MAE) de 4.46281, es el valor medio de los residuos. El estadístico Durbin-Watson (DW) examina los residuos para determinar si hay alguna correlación significativa basada en el orden en el que se han introducido los datos en el fichero.

\section{Discusión}

Resulta relevante mencionar que la ecotoxicología está experimentando un creciente desarrollo con el fin de proporcionar la 
información y el conocimiento básico para la identificación y valoración de la peligrosidad de compuestos (Bro-Rasmussen, 1997; Silveira \& Oliveira-filho, 2013). Durante la década de los años 70 y como resultado de un nuevo paradigma los países industrializados adoptan un esquema de trabajo que permite incorporar esta información recopilada en un proceso de regulación conocido como evaluación de riesgo (Crane, Boxall \& Barrett, 2009).

En este estudio se observó, que la ecuación del modelo cinético-matemático indicó que a muy bajas concentraciones de cianuro libre $(0.024 \mathrm{ppm})$ y en un tiempo relativamente corto (20 minutos), los ejemplares de Gambusia punctata experimentaron la muerte celular de manera que este elemento presentó efectos de letalidad y a medida que dicha concentración va aumentando, el riesgo para los individuos de experimentación es mayor, por cuanto la toxicidad es tan letal que provocó el $100 \%$ de muerte a los individuos, siendo dicho resultado interpretado como muy significativo.

En un estudio realizado por G. Argota, H. Argota y Mamani (2014), sobre cianuro libre en efluentes mineros se encontraron concentraciones promedios entre $0.13 \mathrm{y}$ $0.14 \mathrm{ppm}$, concluyéndose que los vertimientos presentaron concentraciones muy superiores a lo permisible, pudiendo ocasionar efectos ambientalmente. En este caso, dichos efluentes finalmente son descargados en las aguas superficiales del río Ananea (pendiente), el cual cambia su nombre por Ramis en su curso bajo.

En otra investigación, realizada por Argota, Espinoza y Iannacone (2015), encontraron que al evaluar la toxicidad letal media por exposición a cianuro libre en efluentes y relaves mineros utilizando Brachydanio rerio y Eisenia andrei como biomodelos se observó, una mortalidad del 100\% de los individuos de Brachydanio rerio a las 24 horas de duración del ensayo, cuya concentración letal media correspondió a $0.078 \mathrm{mg} / \mathrm{L}^{-1}$, mientras que en Eisenia andrei la concentración letal media ocurrió a las 48 horas de duración del ensayo y correspondió a $0.016 \mathrm{~g}$ de relave. En esta investigación, se concluyó que los bioensayos indicaron efectos de toxicidad inmediata en efluentes y relaves mineros expuestos a cianuro libre.

En comparación con los resultados obtenidos en el presente estudio, puede considerarse que las concentraciones de cianuro libre que son descargadas por efluentes mineros, al parecer no solo que carecen de tratamientos, sino que presentan movilidad y persistencia ambiental, pues aun cuando pudieran disminuir sus concentraciones por efectos de dilución al mezclarse con las aguas del cuerpo receptor, se observó que al 83\%, aproximadamente, (con relación a $0.14 \mathrm{ppm}$ ) de dicha dilución se observaron efectos de toxicidad inmediata.

Considerando lo anterior, si bien es cierto que los gobiernos están adoptando medidas con carácter normativo, así como determinadas políticas para minimizar los riesgos ambientales y a la salud pública, puede mencionarse que todavía faltan esfuerzos relacionados con la gestión científica para la búsqueda de mecanismos reguladores más eficientes, pues si bien es cierto que la evaluación de riesgo es una herramienta científica de vital aplicación (Schowanek et al., 2001), aún se necesita la incorporación de variables en forma más precisa e integral conjuntamente con acciones simultáneas para poder considerar la estimación de efectos e impactos por exposición a cargas contaminantes con la mayor precisión y exactitud posible.

Finalmente, la evaluación de riesgo ecológico es un proceso a través del cual se pretende estimar la probabilidad de que ocurran, en el futuro, o estén ocurriendo en el presente, efectos ecológicos adversos en un ecosistema focal como resultado de la exposición a uno o más agentes estresantes, liberados al 
PREDICCIÓN DE RIESGO ECOTOXICOLÓGICO DADA LA EXPOSICIÓN A CIANURO LIBRE MEDIANTE MODELACIÓN CINÉTICO-MATEMÁTICA EN CONDICIONES CONTROLADAS UTILIZANDO EL BIOMOTOR GAMbuSia PunCTATA

ambiente por la actividad antrópica (Encina \& Díaz, 2001; US EPA, 1992).

En tal sentido, la base para medir el riesgo es igual al dańo potencial por la exposición y depende de la cuantificación del efecto ecotoxicológico y de la expresión de la toxicidad formulada en términos de dosis, concentración y de efecto letal para el $50 \%$ de los individuos, así como el máximo aceptable de concentración del tóxico, nivel más bajo de efecto observable (LOEC), además del nivel más bajo de concentración no observable (NOEC) (Bakhtiar, 2008; Garte \& Bonassi, 2005), en que en este estudio, fue utilizado el indicador de concentración letal media (50\%), empleando una especie reconocida como biomonitor ecotoxicológico de ambientes naturales (Argota, Iannacone \& Fimia, 2013), lo cual indicó mayor probabilidad de riesgo para el valor de uso de las aguas.

\section{Referencias}

Argota, G., Argota, H. \& Mamani, J. (2014). Determinación analítica por exposición a cianuro libre en efluentes mineros, planta artesanal Poderosa-Ananea. Cátedra Villarreal, 2(1), 11-18.

Argota, G., Espinoza, N. \& Iannacone, J. (2015). Evaluación de la toxicidad letal media por exposición a cianuro libre en efluentes y relaves mineros utilizando los biomodelos Brachydanio rerio y Eisenia andrei. Cátedra Villarreal, 3(1), 83-88.

Argota, G., Iannacone, J. \& Fimia, R. (2013). Características de Gambusia punctata (Poeciliidae) para su selección como biomonitor en ecotoxicología acuática en Cuba. The Biologist, 11(2), 229-236.

Bakhtiar, R. (2008). Biomarkers in drug discovery and development. Journal of Pharmacological and Toxicological Methods, 57, 85-91.

Bro-Rasmussen, F. (1997). The environmental experience: ecosystem protection. Archives of Toxicology, 19, 155-166.

Carpenter, R. A. (1995). Risk assessment. Impact Assessment, 13, 153-187.

Crane, M., Boxall, A. B. A. \& Barrett, K. (2009). Veterinary Medicines in the environment. Pensacola, Florida: CRC Press.
Darbra, R. M., Eljarrat, E. \& Barceló, D. (2008). How to measure uncertainties in environmental risk assessment. Trends in Analytical Chemistry, 27(4), 377-385

Van der Oost, R., Beyer, J. \& Vermeulen, N. P. (February, 2003). Fish bioaccumulation and biomarkers in environmental risk assessment: a review. Environmental Toxicology and Pharmacology, 13(2), 57-149.

Encina, F. \& Díaz, O. (2001). Estimación del riesgo ecológico y protección asociado a algas bentónicas marinas. En K. Alvear \& T. Antezana (Eds.), Sustentabilidad de la biodiversidad (pp. 336-357). Chile: Universidad de Concepción.

Evans, J. S. (2002). Introduction to risk analysis [Slides]. Second course on air quality management (MIT-CAM).

Frey, H. C. (1992). Quantitative analysis of uncertainty and variability in environmental policy making. Recuperado de http://www4.ncsu. edu/ $\sim$ frey/reports/frey_92.pdf

Garte, S. \& Bonassi, S. (November, 2005). Linking toxicology to epidemiology: biomarkers and new technologiesSpecial issue overview. Mutation Research, 592(1), 3-5. 
Hogarth, R. M. (2006). Los seguros y la seguridad después del 11 de Septiembre: ¿Acaso el mundo se ha vuelto un lugar más "riesgoso"? Recuperado de: http://www. cholonautas.edu.pe/modulo/upload/ Segur.pdf

International Organization for Standardization. (1980). Normalization Standard International: ISOa 5667-1. Water quality. Part 1: Guidance on the design of sampling programmes.

International Organization for Standardization. (1991). Normalization Standard International: ISOb 5667-2. Water quality. Part 2: Guidance on sampling techniques.

International Organization for Standardization. (1994). Normalization Standard International: ISOc 5667-3. Water quality. Part 3: Guidance on the preservation and handling of samples.

Lee-Steere, C. (2009). Environmental risk assessment guidance manual for industrial chemicals. Australia: Environment Protection and Heritage Council.

Litchfied, J.T. Jr. \& Wilconxon, F. (1949). A simplified method of evaluation dose-effect experiments. Journal of Pharmacology and Experimental Therapeutics, 96(2), 99-113.

Management Programme for the Gorgon Development: MPGD. (2004). Risk assessment process. In autor, Environmental Impact Statement/Environmental Review and Management Programme for the Gorgon Development (pp. 288301). Recuperado de https://www. chevronaustralia.com/docs/defaultsource/default-document-library/ gorgon_ch11_lr.pdf?sfvrsn=0

Mongomery D. C. (1991). Diseño y Análisis de Experimentos. México D.F.: Grupo Editorial Iberoamérica S.A.
Morgan, M. \& Henrion, M. (1990). Uncertainty: A guide to dealing with uncertainty in quantitative risk and policy analysis. New York: Cambridge University Press.

Montico, S. \& Di Leo, N. (2015). Riesgo ambiental por pesticidas en una cuenca del sur de la provincia de Santa Fe, Argentina. Rev. Int. Contam. Ambient., 31(2), 165-172.

National Academy of Sciences: NAS. (1983). Risk assessment in the federal government: managing the process. Washington DC: National Academy Press.

Organización Europea para la Cooperación y el Desarrollo. (1992). OECD Guidelines for testing of chemicals. Fish, acute toxicity test (Test No. 203). Paris, Francia: OECD Publishing.

Rao, S., Chirkov, V., Dentener, F., Van Dingenen, R., Pachauri, S., Purohit, P., .. Schoepp, W. (2012). Environmental modeling and methods for estimation of the global health Impacts of air pollution. Environmental Modeling \& Assessment, $17(6), 613-622$.

Samantray, P., Mishra, B. K., Panda, C. R. \& Rout, S. P. (2009). Assessment of water quality index in Mahanadi and Atharabanki rivers and Taldanda canal in Paradip Area, India. Journal of Human Ecology, 26(3), 153-161.

Statgraphics Plus (Versión 5.1.) [Software de computación]. Virginia, Estados Unidos: Statpoint Technologies, Inc.

United Nations Environment Programme \& International Programme on Chemical Safety (1999). Environmental Risk Assessment. In Author, Chemical risk assessment. Human risk assessment, environmental risk assessment and ecological risk assessment (pp.107-176). Suiza: WHO.

United States Environmental Protection Agency: US EPA. (1998). Guidelines for ecological risk assessment (EPA/630/ R-95/002F). Washington DC. 
United States Environmental Protection Agency: US EPA. (2001). Risky Business? An overview of risk assessment and RCRA (EPA530-F-00-032). Washington DC.

United States Environmental Protection Agency: US EPA. (2002). Methods for measuring the acute toxicity of effluents and receiving waters to freshwater and marine organisms ( $5^{\text {th }}$ ed., EPA821-R-02-012. 266). Washington DC.

Schowanek, D., Fox, K., Holt, M., Schroeder, F. R., Koch, V., Cassani, G., \& Feijtel, T.C. (2001). GREATER: a new tool for management and risk assessment of chemicals in river basins. Contribution to GREAT-ER \# 10. Water Science and Technology, 43(2), 179-185.

Whyte, A. V. \& Burton, I. (1980). Environmental Risks. En Autor, Environmental Risk Assessment. Recuperado de http://dge. stanford.edu/SCOPE/SCOPE_15/ SCOPE_15.html

World Bank Group, United Nations Environment Programme \& the United Nations Industrial Development Organization. (1999). Pollution prevention and abatement Handbook 1998. Toward Cleaner Production. Washington DC: World Bank Group. 
